Livraisons

d'Histoire

de l'Architecture

\section{Livraisons de l'histoire de l'architecture}

$26 \mid 2013$

Les ministres et les arts

\title{
L'abbé Terray, seigneur de La Motte-Tilly
}

L'abbé Terray, seigneur of La Motte-Tilly

\section{Rose-Marie Chapalain}

\section{CpenEdition}

Journals

Édition électronique

URL : http://journals.openedition.org/lha/326

DOI : $10.4000 /$ /ha. 326

ISSN : 1960-5994

\section{Éditeur}

Association Livraisons d'histoire de l'architecture - LHA

Édition imprimée

Date de publication : 10 décembre 2013

Pagination : 43-61

ISSN : 1627-4970

\section{Référence électronique}

Rose-Marie Chapalain, "L'abbé Terray, seigneur de La Motte-Tilly », Livraisons de l'histoire de

l'architecture [En ligne], 26 | 2013, mis en ligne le 10 décembre 2015, consulté le 30 avril 2019. URL:

http://journals.openedition.org/lha/326 ; DOI : 10.4000/lha.326 


\section{L'ABBÉ TERRAY, SEIGNEUR DE LA MOTTE-TILLY}

Le château et les jardins de la Motte-Tilly, résidence de Joseph-Marie Terray de 1748 à son décès en 1778, rouverts au public depuis mars 2013 suite à d'importants travaux électriques ${ }^{1}$, permettent d'aborder la question du contrôleur général des finances de Louis XV sous l'angle de sa relation avec les arts ${ }^{2}$. L'élaboration d'une étude monographique du château de la Motte-Tilly et de ses jardins a été rendue difficile par l'absence de biographie et l'existence de peu d'études consacrées à Joseph-Marie Terray. Quelques-unes, historiques, traitent le personnage par le biais de ses fonctions : contrôleur général des finances et directeur général des bâtiments du roi ${ }^{3}$. Cette dernière facette a été abordée par Colin B. Bailey, dans de nombreux articles concernant la dernière période de la vie de Joseph-Marie Terray ${ }^{4}$. L'étude monographique de la Motte-Tilly, effectuée dans le cadre des travaux de master $^{5}$, a permis de traiter certains aspects de la vie du contrôleur général des finances avant 1760 , mais également les travaux menés aux limites de la généralité de Paris ${ }^{6}$. Le château du XVIII ${ }^{e}$ siècle a été remanié partiellement par les propriétaires qui succédèrent à Joseph-Marie Terray ${ }^{7}$ avant d'être légué à la Caisse nationale des Monuments historiques et des Sites par la marquise de Maillé en $1972^{8}$

1. Inauguration du château le 26 mars 2013 . Le château a été partiellement fermé pendant une période de deux ans pour des travaux allant de la menuiserie à l'électricité.

2. Magalie Quintard-Lenoir, "Joseph-Marie Terray abbé de cour et ministre fastueux ", Les ministres des arts, Christophe Morin dir., colloque organisé les 3 au 4 juillet 2012 à Tours et Blois, à parâtre.

3. Lucien Laugier, Un ministère réformateur sous Louis XV: le Triumvirat (1770-1774), Paris, La Pensée Universelle, 1975, 656 p.

4. Colin B. Bailey, Patriotic taste, collecting modern art in pre-revolutionary Paris, London, Yale University Press, 2002, p. 71-100 et "Portraits of an abbé : towards an interpretative iconography of Joseph-Marie Terray (1715-1778) ", La Gazette des Beaux-Arts, septembre 1994, p. 24-38.

5. Rose-Marie Chapalain, Le château et les jardins de la Motte-Tilly de 1748 à 1796 dans l'Aube, mémoire de master 2 sous la direction de Dominique Massounie, université Paris Ouest-La Défense, 2013.

6. Le premier chapitre du mémoire de master traite du contexte de l'achat du domaine de la MotteTilly ainsi que de la construction du château à partir de 1754. De 1748 à 1754, Joseph-Marie Terray est alors clerc au Parlement de Paris et son frère Pierre Terray de Rosières est maitre des requêtes à la Cour des Aides. Jean-Baptiste-Louis Cocquereau, Mémoires de l'abbé Terray, contrôleur général des finances avec une relation de l'émeute arrivée à Paris en 1775, et suivis de quatorze lettres d'un actionnaire de la Compagnie des Indes, Londres, 1776, p. 2.

7. Antoine-Jean Terray est propriétaire de 1780 à 1794 , Claude-Hippolyte Terray jusqu'en 1849 , Charles-Louis Terray de Vindé jusqu'en 1866, Charles-Gérard de Rohan-Chabot de 1910 jusqu'en 1964 et enfin Aliette de Rohan-Chabot, épouse de Maillé, jusqu'en 1972.

8. Codicille au testament de la marquise de Maillé, du 11 décembre 1971, Charles de Cossé-Brissac, "Le château de la Motte-Tilly ", La Sauvegarde l'Art français, cahier 1, 1979, p. 185-191. 
et ouvert au public en $1978^{9}$. Le domaine est aujourd'hui constitué d'éléments composites : des aménagements du XIX ${ }^{\mathrm{e}}$ siècle sont mêlés à des bâtiments du début du XXe siècle et du XVIII ${ }^{e}$ siècle. Les bâtiments forment un ensemble homogène et cohérent, mais dont la lecture historique est ainsi rendue difficile. On tâchera ici de proposer des informations complémentaires et inédites sur l'histoire de l'édifice ainsi qu'une étude architecturale permettant de comprendre les choix du propriétaire et de l'architecte, ainsi que la place du château au sein de la carrière de Joseph-Marie Terray.

\section{Les archives de la Motte-Tilly: un fonds important}

Le fonds d'archives du château de la Motte-Tilly, propriété du Centre des Monuments nationaux a fait l'objet d'un dépôt au début des années 2000 aux archives départementales de l'Aube ${ }^{10}$. Le fonds est classé de la manière suivante: les archives personnelles, professionnelles et familiales des propriétaires du château d'un côté, et les archives seigneuriales et domaniales de l'autre. Les dossiers sont classés par famille, par ordre d'importance et par ordre chronologique. Dans les familles ayant possédé les seigneuries et les familles alliées, on retrouve celles des Le Nain, Paignon-Dijonval ou encore Rouen des Mallets. L'articulation se fait autour de trois familles principales: les familles Terray, Morel-Vindé et RohanChabot. Ensuite vient la partie domaniale et seigneuriale : le domaine de la MotteTilly et les domaines de Vindé et de Courtavant. Le classement de ces documents se fait suivant les seigneuries et les dépendances du château champenois. Ensuite s'y ajoute la comptabilité de ces différents domaines, divisée en deux sous-ensembles : les propriétés parisiennes de la famille Terray et leurs régies. Les archives départementales de l'Aube conservent aussi les minutes notariales de la Motte-Tilly et du domaine de Rosières de 1768 à $1786^{11}$. Les séries $E^{12}$ et $C^{13}$ ainsi que le fonds

9. Ibid., p. 186 .

10. Le fonds, $144 \mathrm{~J}$, est composé de 1264 dossiers, ce qui représente environ 127 mètres linéaires.

11. Arch. dép. Aube, $2 \mathrm{E} 66 / 1$ et $2 \mathrm{E} 66 / 2$. Ces minutes montrent la multiplication des baux, notamment effectués par l'abbé Terray et son neveu pour engager de la main d'œuvre pour travailler sur les terres de la Motte-Tilly. À cela s'ajoutent les minutes notariales de la ville de Nogent-sur-Seine et d'Avant-lès-Marcilly, où l'on trouve quelques baux relatifs à Pierre Terray de Rosières, le frère de l'abbé Terray pour le domaine de Rosières.

12. Arch. dép. Aube, E E/1/1099 : la famille Terray à Gumery et à Villenauxe; E/1034 : l'accord relatif à ces dômes fait entre le curé d'Avant et Pierre Palluau, fils du précédent conseiller au Parlement de Paris (1717); l'approbation de cet acte par le curé et Pierre Terray, conseiller au Parlement et François Terray, seigneur d'Avant et de Rosières (1741) ; E/1045 : actes terriers dont ceux AntoineJean Terray (1788).

13. Arch. dép. Aube, $\mathrm{C} / 2104$ : bail par la ville d'un terrain longeant le canal créé par l'abbé Terray. La ville représentée par Nicolas du Mez, conseiller du roi, président en l'élection, maire ; Étienne Simon Lefebvre, premier échevin ; Rémy Thomas, échevin ; C/2106 : correspondance entre le contrôleur Terray et l'intendant de Paris concernant de nouvelles réparations au collège; C/2156: procès-verbal d'arpentage des terres de la Motte-Tilly, 1783-1788. 
d'archives du Prince Xavier de Saxe ${ }^{14}$ apportent des informations relatives au domaine de la Motte-Tilly et à la famille du propriétaire.

Enfin, les archives départementales de l'Aube conservent également un ensemble de plans du château champenois pour la période qui nous concerne. On dénombre huit plans du château et des jardins, dont un projet pour la distribution du rezde-chaussée daté de $1754^{15}$, un plan de 1755 signé par l'architecte FrançoisNicolas Lancret ${ }^{16}$ et deux plans où sont représentés les jardins, la Seine et la route. Le plan de 1755 représente les terrasses, quelques bosquets et quinconces près du château. Ceux de $1770^{17}$ et de $1780^{18}$ figurent l'intégralité des jardins. Le premier présente le domaine avant la construction du canal et le second, après. Un dessin de F. de La Brunière reproduit les jardins de la Motte-Tilly depuis l'extrémité du canal ${ }^{19}$. Enfin, un plan de distribution du rez-de-chaussée des différents bâtiments de 1782 est également conservé ${ }^{20}$. Trois autres plans s'ajoutent dans cette décennie, réalisés à l'occasion des travaux de réaménagements par Antoine-Jean Terray ${ }^{21}$. En revanche, il n'existe pas de représentation de l'élévation du château avant celle de 1774, exécutée par F. de La Brunière, et les seuls plans pour les niveaux supérieurs datent des années 1780 .

Le minutier central des notaires des Archives nationales regorge de documents inédits. Un dépouillement systématique des études proches du Palais-Royal et des notaires ayant fait des transactions avec les personnes concernées a été réalisé pour les années 1745 à 1757 . L'acte de vente du domaine de la Motte-Tilly ainsi que des minutes récapitulant les transactions réalisées ont été mis au jour ${ }^{22}$.

14. Arch. dép. Aube, EE 001513 : correspondance pårticulière du prince Xavier de Saxe avec la princesse Christine de Saxe, 1770 ; EE 001608 : correspondance particulière avec Jean-Baptiste Rivière, conseiller de la légation de l'électeur de Saxe à Paris, 1769 et EE 001631 : correspondance particulière avec Joseph-Marie Terray, 1774.

15. Arch. dép. Aube, $144 \mathrm{~J} 873$ : [François-Nicolas Lancret], projet du plan au rez-de-chaussée d'un château à bâtir à la terre de la Motte-Tilly près de Nogent-sur-Seine, 1754, 150 sur $100 \mathrm{~cm}$, plume et rehaussé à l'aquarelle.

16. Arch. dép. Aube, 144 J 1469 : [François-Nicolas Lancret], plan au rez-de-chaussée du château et des dépendances de la Motte-Tilly, 1755, 120 sur $127 \mathrm{~cm}$, dessin à la plume et lavis.

17. Arch. dép. Aube, $144 \mathrm{~J} 837$ : [Anonyme], plan du château et des jardins de la Motte-Tilly, [avant 1770], 108 sur $171 \mathrm{~cm}$, plume et lavis.

18. Arch. dép. Aube, $144 \mathrm{~J} 886$ : [Anonyme], plan du château et des jardins de la Motte-Tilly, non daté [avant 1780], 168 sur $116 \mathrm{~cm}$, dessin à la plume et aquarelle.

19. Une gravure de N. Ponce représentant le Cap-Français d'après le dessin F. de la Brunière dans Pierre de Vaissière, Saint-Domingue (1629-1789): la société et la vie créole sous l'Ancien Régime, Paris, Perrin, 1909, p. 331.

20. Arch. dép. Aube, $144 \mathrm{~J} 878$ : [Anonyme], plan du rez-de-chaussée du château et des dépendances de la Motte-Tilly, 1782, 150 sur $98 \mathrm{~cm}$, dessin à la mine de plomb rehaussé à l'aquarelle.

21. Arch. dép. Aube, 144 J 876 : [Jean-Benoît-Vincent Barré], Plans du rez-de-chaussée, du premier étage et du second étage, [1784], 113 sur $72 \mathrm{~cm}$, plume et lavis.

22. Arch. nat., M.C./XXIII/559 : quittance de paiement, 5 septembre 1748 ; Arch. nat., M.C./CXVI/ 333 : vente entre le duc de Noailles et le seigneur de Fulvy, 24 août 1748. 


\section{Le contexte d'achat du domaine de la Motte-Tilly en 1748}

L'étude de ce fonds d'archives a permis de rétablir un certain nombre d'erreurs rapportées dans la notice historique de Charles-Paul Léger ${ }^{23}$. On retient que le domaine de la Motte-Tilly a appartenu à un certain nombre de familles nobiliaires $^{24}$ avant d'acquérir le statut de comté au début du XVIII ${ }^{\mathrm{e}}$ siècle $^{25}$. En 1743 , la famille Terray s'installe dans la Champagne méridionale en faisant l'acquisition des domaines d'Avant et de Rosières ${ }^{26}$, aujourd'hui la commune d'Avant-lèsMarcilly ${ }^{27}$. L'achat réalisé par les frères Terray s'inscrit dans la continuité de l'entreprise de leur oncle, François Terray de Rosières, premier médecin de la princesse Palatine ${ }^{28}$. Ils font l'acquisition auprès du duc Maurice de Noailles des domaines de la Motte-Tilly, de Gumery et de La Pré d'Athis, le 24 août 1748 par l'intermédiaire de Jean-Henri-Louis Orry de Fulvy ${ }^{29}$. Ce dernier, demi-frère de Philibert Orry, contrôleur général des finances, commence en 1723 par intégrer le Parlement de Paris comme conseiller en 1723 pour devenir en 1731 maître des requêtes, commissaire du roi auprès de la Compagnie des Indes en 1733, intendant des finances en 1737-1751, et enfin, maitre des requêtes honoraire en 1738. Il hérite du domaine de la Chapelle-Godefroy, à la mort de son demi-frère le 9 novembre 1747 et grâce à la vente proposée par le duc de Noailles, récupère une partie du comté de Nogent pour compléter celle que son père avait acquise en $1697^{30}$. Quant à la seigneurie de la Motte-Tilly, elle perd son rang comtal ${ }^{31}$.

23. Charles-Paul Léger, "Notice sur l'ancienne seigneurie et l'ancien comté de la Motte-Tilly ", Mémoires de la société académiques de l'Aube, t. LXXVII, Troyes, Société académique de l'Aube, 1923, p. 3-120.

24. Charles-Paul Léger réalise une notice historique sur le château de la Motte-Tilly et écrit sur les différents seigneurs qui en ont été les propriétaires depuis le début du XVe siècle : Pierre des Essarts, Henri de Pouligny, la famille Raguier, la famille d'Elbeyne, le duc de Bournonville, la famille de Noailles et la famille Terray.

25. Charles-Paul Léger, "Notice sur l'ancienne seigneurie et l'ancien comté de la Motte-Tilly ", op. cit., p. 82-83.

26. Arch. dép. Aube, 144 J 7 : titres des terres de Rosières, d'Avant et de Vouarce de François Terray de Rosières, 1743.

27. Le domaine de Rosières est signalé sur la carte de Cassini, Nogent-sur-Seine et ses environs, avant 1756. Ce domaine se situe au sud de Nogent-sur-Seine et à proximité de la Chapelle-Godefroy. Il touche également le domaine de la Motte-Tilly.

28. Jean-Baptiste-Louis Cocquereau, Mémoires de l'abbé Terray, contrôleur général des finances avec une relation de l'émeute arrivée à Paris en 1775, et suivis de quatorze lettres d'un actionnaire de la Compagnie des Indes, Londres, 1776, p. 2.

29. Arch. nat., M.C./CXVI/333 : vente concernant le maréchal-duc de Noailles et monsieur Orry de Fulvy, suivie de la déclaration et de la reconnaissance, 24 août 1748.

30. Françoise Bayard, Joël Félix et Philippe Hamon, Dictionnaire des surintendants et contrôleurs généraux des finances, $X V I^{e}, X V I I^{e}, X V I I I^{e}$ siècles, Paris, Comité pour l'histoire économique et financière de la France, 2000, p. 138.

31. Charles-Paul Léger, "Notice sur l'ancienne seigneurie et l'ancien comté de la Motte-Tilly", op. cit., p. 83. 
Le document qui suit est intitulé "Déclaration et reconnaissance ». L'acquéreur déclare que les bénéficiaires de cet achat sont Pierre Terray de Rosières et JosephMarie Terray, qui se partagent à part égale les biens, soit les terres de la MotteTilly, la seigneurie de la Pré d'Athis, les seigneuries de Fontenay, de Guimery et de Boissery. Quant au seigneur de Fulvy, il conserve le comté de Nogent-sur-Seine. Vient ensuite la partie financière; l'acte de vente mentionne la somme de 330000 livres pour l'ensemble des domaines vendus ${ }^{32}$. Les frères Terray paient la somme de 260000 livres $^{33}$. Le document notarié fait également mention des terres de Mâcon et de la seigneurie des Ormeaux, à proximité du domaine de Rosières. L'achat d'un domaine à l'extérieur de Paris et la construction d'un château et d'un jardin nouveau représentent un engagement financier considérable motivé par une véritable stratégie sociale. Ils avaient déjà hérité des biens de leur oncle, François Terray de Rosières, décédé en $1747^{34}$ et Pierre Terray de Rosières avait acheté en 1743 la charge de procureur général à la Cour des Aides de Paris avant de celle de maître des requêtes au Conseil en 1749 . Construisant sa carrière, il avait épousé en 1743 Marie-Félicité Le Nain, la fille d'un intendant du Languedoc ${ }^{35}$. Les armoiries représentées sur les façades du château témoignent de cette union. Pourtant la place de Pierre Terray de Rosières dans la gestion du domaine et dans la construction de l'édifice resta secondaire ${ }^{36}$. Le principal acteur de ces embellissements se révèle être son frère, l'abbé Joseph-Marie Terray. La présence de ce dernier sur les livres de comptes des années 1755 à 1758 témoigne d'un grand investissement personnel. C'est en effet lui qui valide les mémoires et donne son accord pour le paiement des ouvriers et des domestiques ${ }^{37}$.

32. Ibid., note 28 : "Sieurs Terray frères, comme ils s'y obligent solidairement pour leur renonciation de droit requise de garanties mon dit Sieur de Fulvy de tous les engagements qu'il a pris par ledit contrat relativement aux dites terres acquises à leur profit et attendu que le prix total de toutes lesdites terres ensemble ont la somme de 330000 livres."

33. Ibid., note 28 : "Mes dits Sieurs de Fulvy et Terray frères reconnaissent que mon dit Sieur de Fulvy en a dû payer pour ledit comté de Nogent et dépendances celle de soixante-dix mille livres et mes dits Sieurs Terray frères celle de 260000 livres pour lesdites terres et seigneurie de la MotteTilly, le Pré d'Athis, Gumery et Fontenay, Boissery. "

34. Arch. nat., M.C./XVII/12 : testament de François Terray de Rosières, 3 août 1747. Dans le même carton, se trouve le document qui aborde les questions de la succession de François Terray datant du 31 décembre 1753 et le partage entre Joseph-Marie Terray et Pierre Terray de Rosières, le 28 février 1754 .

35. Il se marie le 23 avril 1743 , à la paroisse Saint-Roch, avec Renée-Félicitée Le Nain, fille de Jean Le Nain VII (1698-1750) qui est maître des requêtes en 1726, intendant de Poitiers en 1731 puis du Languedoc en 1743 et est conseiller d'État à partir de 1748. François-Alexandre Aubert de la Chenaye Desbois, Dictionnaire de la noblesse : contenant les généalogies, l'histoire et la chronologie des familles nobles de France, Paris, 1863-1876, p. 661.

36. Arch. dép. Aube, $144 \mathrm{~J} 751$ : les livres de comptes du domaine de la Motte-Tilly, de 1755 à 1758 répertorient les différentes transactions par Mr. Bossuat, régisseur du domaine. Les transactions sont systématiquement validées par " Mr. Terray ", la première mention de "Mr. Terray de Rosières " date de 1758.

37. Ibid. 


\section{Le château de la Motte-Tilly: une maison de campagne ${ }^{38}$ ?}

En 1748, messieurs Terray de Rosières ont acquis la terre de la Motte-Tilly, Courceroy, Gumery et Fontenay de monsieur le Maréchal de Noailles, ont fait démolir toute la couverture du château de la Motte-Tilly sur la rivière de la Seine avant de vendre la terre. Messieurs Terray de Rosières, acquéreur de ladite terre ont continué la démolition et au printemps 1754 ont fait jeter un plan d'un nouveau château sur la hauteur du chemin de Nogent, auquel on a donné le nom de château de Bellevue. La première pierre a été posée le 25 juin de la susdite année 1754 par madame de Rosières, épouse de haut et puissant seigneur du lieu, procureur général de la Cour des Aides ${ }^{39}$.

\section{La maison de campagne et son mobilier}

D'après les plans et relevés retrouvés, l'ancien château champenois était situé au bord de la Seine et près du village. Un chemin traversait ce village : signe d'une relation privilégiée avec l'église, mais aussi d'un lien fort avec les habitants. Le château se présentait sous la forme d'un quadrilatère flanqué de tours à ses angles ${ }^{40}$. Le registre paroissial du village voisin, Courceroy, témoigne de la transaction entre les frères Terray et le duc de Noailles, mais également de l'état dans lequel se trouve le château en 1748 : il est en partie en ruine. Les nouveaux propriétaires décident alors de poursuivre la démolition de l'ancienne demeure des seigneurs de la MotteTilly et de construire un nouvel édifice. Le projet de 1754 et le plan de 1755 (ill. 1) montrent une rupture évidente avec cette dimension du château " féodal " ${ }^{41}$. L'espace environnant le château y est représenté, mais il s'agit d'un jardin ordonné avec des terrasses, des bosquets, des topiaires, des allées et des boulingrins. Il s'agit plutôt d'un jardin de plaisance. Les frères Terray décident de faire construire sur les hauteurs de la Motte-Tilly. Comme le conseille Jacques-François Blondel, le meilleur emplacement est "une éminence d'où l'on puisse découvrir le grand nombre d'objet qu'il sera possible, sans que néanmoins elle soit trop en prise au vent du nord ${ }^{42}$ ". Le plan de François-Nicolas Lancret permet de comprendre que la volonté des nouveaux propriétaires est d'ordonner le château et les dépendances par rapport à un axe principal (Nord-Sud) et par rapport à la route et au fleuve

38. Dénomination tirée du traité de Charles-Étienne Briseux, L'Art de bâtir des maisons de campagne, publié chez Prault père à Paris en 1743.

39. Arch. dép. Aube, 135 G 3 : l'extrait provient des registres paroissiaux de l'église Saint-Pierre-etPaul de Courceroy, 1668-1792, cité dans Franck Gérard, "Le château de la Motte-Tilly et les travaux de 1780 ", La Vie en Champagne, hors-série 2012, Troyes, p. 9.

40. Arch. dép. Aube, 144 J 874 : François-Nicolas Lancret, Plan de l'ancienne forteresse de la MotteTilly avec son ancien parc et lieux adjacents, mine de plomb, 1754 et une représentation proposée par Vincent Cochet dans Le Château de la Motte-Tilly, Paris, Éditions du Patrimoine, 2005, p. 5.

41. Arch. dép. Aube, $144 \mathrm{~J}$ 873: François-Nicolas Lancret, Plan du rez-de-chaussée du château de la Motte-Tilly, 1755, plume et lavis, $80 \times 120 \mathrm{~cm}$.

42. Jacques-François Blondel, De la distribution des maisons de plaisance et de la décoration en général, 1737-1738, vol. 1, p. 8-9. 


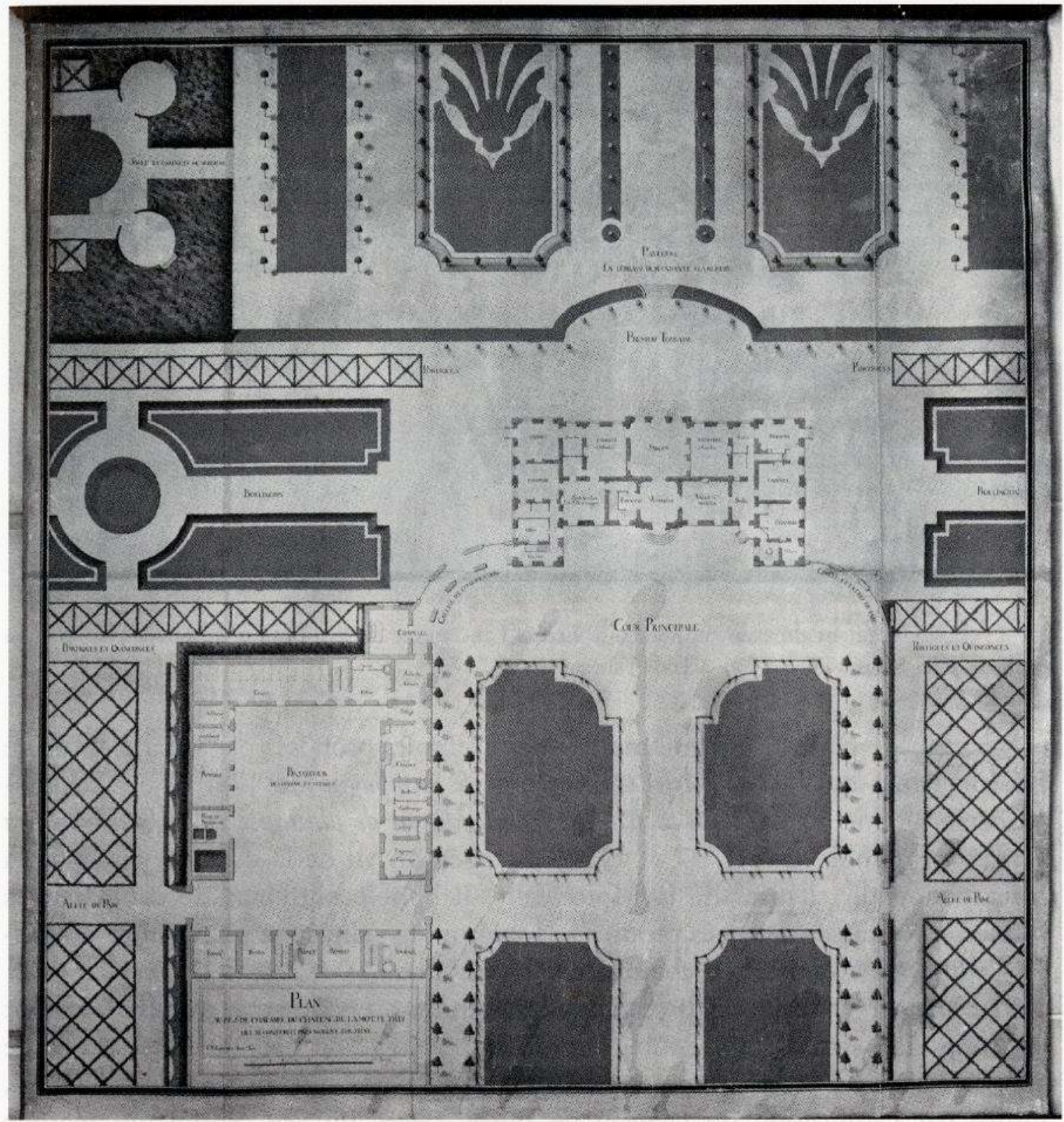

Ill. 1 : François-Nicolas Lancret, "Plan au rez-de-chaussée du château et des dépendances de la MotteTilly ", 1755, dessin à la plume rehausse à l'aquarelle, $120 \times 127 \mathrm{~cm}$. Arch. dép. de l'Aube, $144 \mathrm{~J} 1469$. Cl. de l'auteur.

(ill. 3). Le château s'inscrit bien dans un espace ordonné et l'acquisition de nouvelles terres qui s'effectue parallèlement permet de poser ici l'hypothèse d'un usage "public » du château et de son domaine ${ }^{43}$. Le château de la Motte-Tilly serait un espace de représentation et un instrument d'ascension sociale. L'élévation du château de Rosières, première résidence de la famille Terray dans la région, devait posséder des signes marqueurs de féodalité, ce qui permet de supposer leur absence sur le château de la Motte-Tilly. Ce dernier pourrait ainsi être considéré comme

43. Arch. dép. Aube, 144 J 390 : rentes constituées, rachat par l'abbé Terray, titres, quittances pour rachat, $1^{\text {er }}$ juillet et 26 septembre 1749 . 


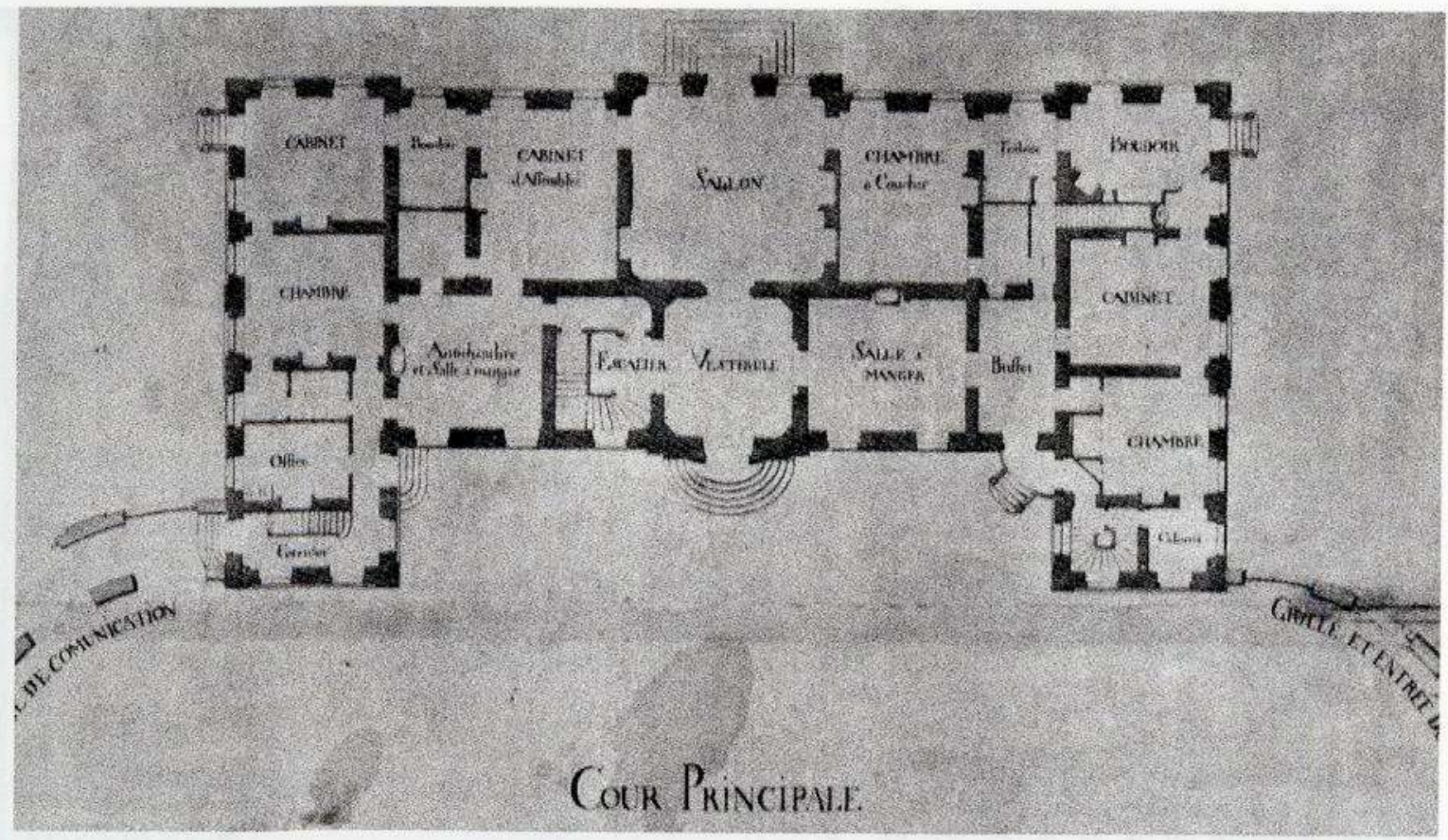

Ill. 2 : Agrandissement du plan de François-Nicolas Lancret sur la distribution du rez-de-chaussée du château de la Motte-Tilly, 1755. Cl. de l'auteur.

un château de plaisance, sur le modèle des exemples proposés par Charles-Étienne Briseux dans son Art de batir des maisons de campagne de $1743^{44}$ ou JacquesFrançois Blondel dans De la distribution des maisons de plaisance et de la décoration en général, publié entre 1737 et 1738 . En effet, au vu de la date du début de la construction du château de la Motte-Tilly, il semble pertinent de comparer les formes et les caractéristiques propres à la maison de campagne pour tenter de classer l'édifice et de mieux en comprendre sa fonction.

Le plan de 1755 (ill. 2) propose la distribution du rez-de-chaussée représentant un corps de logis massé à double profondeur flanqué de deux ailes en retour de 25 sur 11 toises. Elle s'inscrit dans la tradition de la distribution des châteaux, initiée par l'édification du château de Burly ${ }^{45}$ avec un axe central qui marque l'entrée principale de l'édifice, traverse la cour d'honneur et le jardin, en passant par le vestibule et le salon. La distribution intérieure présente quelques changements par rapport au projet initial. On note la présence de deux salles à manger de part et d'autre de l'axe central du corps de logis, avec un buffet. Le couloir central dans la partie ouest est abandonné. Le rez-de-chaussée est composé de trois espaces : l'espace de réception et des espaces réservés aux frères Terray qui se partagent le domaine ${ }^{46}$. Le séjour champêtre s'organise autour de deux pôles: la

44. Charles-Étienne Briseux, L'Art de bâtir des maisons de campagne, Paris, 1743, t. II, planches 139 et 140 .

45. Le château de Dampierre-en-Burly dans le Loiret. Il est construit au début du XVII ${ }^{e}$ siècle.

46. Arch. dép. Aube, 144 J 647 : "Nous, Pierre et Joseph-Marie Terray, avons compté de la recette des registres de l'année, 758 livres et précédents suivant les détails ci-dessus par le résultat duquel compte ladite recette s'est trouvée monter à ladite somme de 3746 livres 17 sols et 6 deniers, laquelle somme nous soussignés, avons partagé en deux parts égales dont chacun de nous avons pris la sienne." 


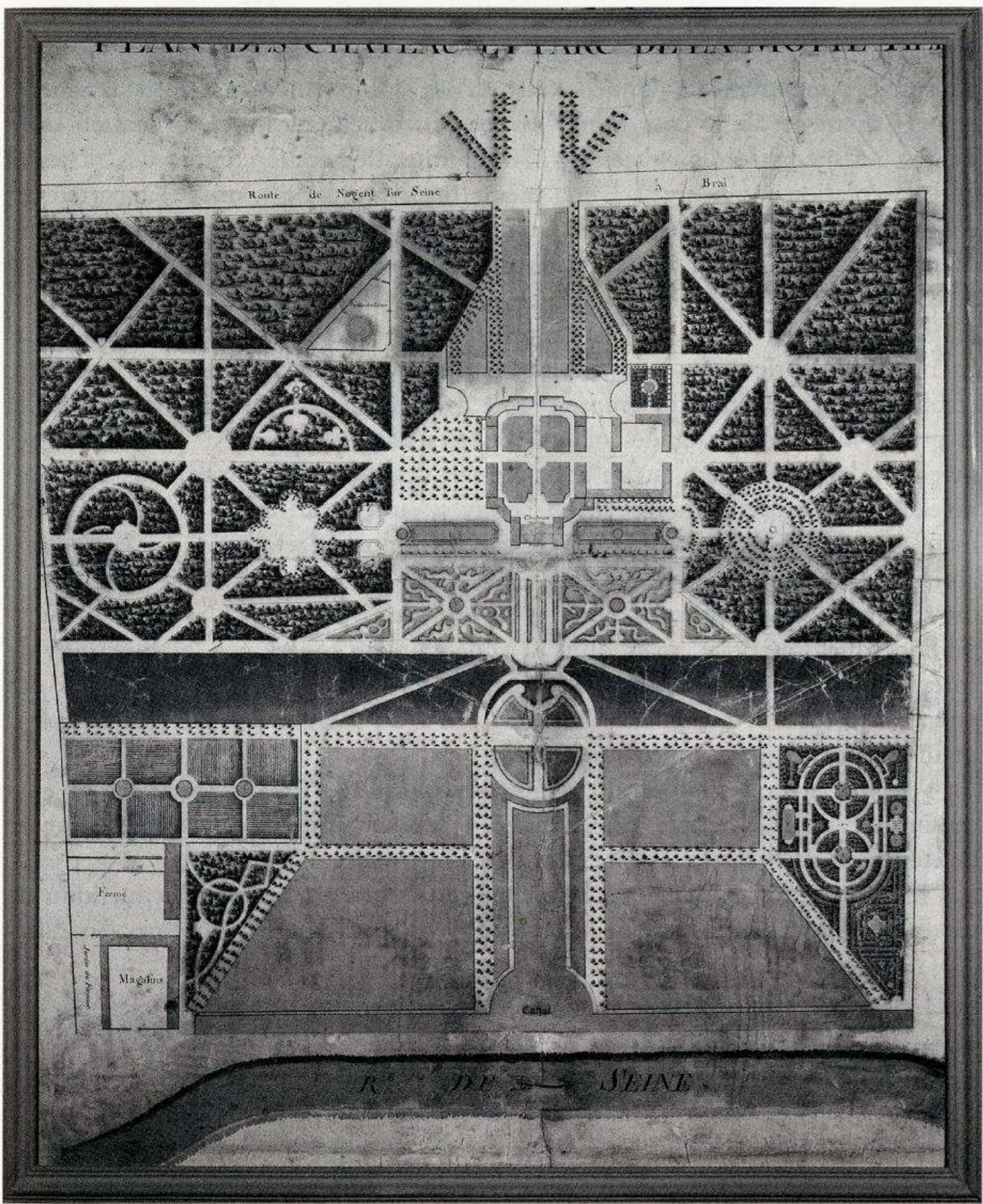

Ill. 3 : Anonyme, Plan du château et des jardins de la Motte-Tilly, non daté [avant 1780], $168 \times 116 \mathrm{~cm}$, dessin à la plume et aquarelle. Arch. dép. de l'Aube, $144 \mathrm{~J} 886$. Cl. de l'auteur.

retraite et la sociabilité. La partie ouest de l'habitat est réservée à Joseph-Marie Terray et la partie est à Pierre Terray, son épouse et ses enfants ${ }^{47}$. Ce niveau est

47. Arch. dép. Aube, 144 J 753 : inventaire des effets et meubles du château de la Motte-Tilly, 1757. Le rez-de-chaussée se compose de trois grands appartements : "l'appartement de monsieur l'abbé ", " l'appartement de Madame " et «l'appartement de Monsieur ». Un quatrième appartement de taille moindre est intitulé l'« appartement des enfants". 
composé de trois grands appartements, d'un vestibule et d'un grand salon, deux salles à manger, d'une salle de billard et d'une salle de compagnie et d'un salon d'hiver. Les appartements donnent tous sur les jardins tandis que les deux salles à manger donnent sur la cour. Nous savons peu de choses sur la décoration intérieure. Un mémoire d'ouvrages de 1781 permet de rendre compte des richesses de la décoration intérieure ${ }^{48}$. De la même manière que le salon et l'appartement d'apparat, la salle à manger devient une pièce de prestige pour les hôtes du maitre de maison. Parmi ces visiteurs, on retrouve le cardinal de Luynes, archevêque de Sens, Mathias Joseph de Barral, évêque de Troyes ${ }^{49}$, mais également deux inspecteurs des Ponts et chaussées ${ }^{50}$. La présence d'une quinzaine d'appartements dans les niveaux supérieurs indique une capacité d'accueil conséquente. Un inventaire établi en 1757, soit peu de temps après la fin des travaux, témoigne également de la qualité du mobilier et des objets déployés dans ces espaces ${ }^{51}$. D'ailleurs cet inventaire ne mentionne aucun appartement destiné aux propriétaires au second niveau. Les appartements des frères Terray se situent au rez-de-chaussée, confirmant une autre caractéristique de la maison de campagne selon Blondel. Cet édifice abrite également du mobilier de jeu ${ }^{52}$. De surcroît, l'inventaire de la bibliothèque du château donne une indication sur les fonctions de l'édifice ${ }^{53}$. Il s'agit d'une bibliothèque très riche, mais dont la collection ne reflète pas les fonctions de Joseph-Marie Terray, ni celles de son frère et de son neveu. La majorité des ouvrages recensés se rattachent à la littérature et au théâtre. Il s'agit bien d'un lieu de divertissement, non un lieu de travail.

Pour parler de l'élévation du château de la Motte-Tilly, il faut se reporter au dessin de F. de La Brunière, réalisé en $1774^{54}$, soit près de vingt ans après la phase de construction. Avant cette date, aucune représentation n'a été conservée à ce jour. Les livres de comptes ainsi que les mémoires d'ouvrages ne font pas mention de modifications importantes dans le corps de logis et la comparaison avec les diffé-

48. Arch. dép. Aube, 144 J 757 : mémoire de Delorme (1781). Il y est décrit un décor qui prend place dans la salle à manger, divisé en deux parties: support du décor et décor. Parmi des objets et matériaux, on note du verre de Bohême, des colonnes de cristal, des branches de fleurs artificielles, quatre groupes de biscuits et des vases avec leurs piédestaux.

49. Charles-Paul Léger, "Notice sur l'ancienne seigneurie et l'ancien comté de la Motte-Tilly ", op. cit., p. 97.

50. Arch. dép. Aube, 144 J 762 : livre de dépenses du domaine de la Motte-Tilly, 1755-1758. Le 2 septembre 1758 : " Mis en dépense lorsque deux inspecteurs de M. [Daniel-Charles] de Trudaine sont venus souper et coucher au château le premier septembre." "

51. Arch. dép. Aube, 144 J 753 : inventaire des effets et meubles du château de la Motte-Tilly, 1757. "Dans le cabinet de monsieur l'abbé, un serre papier avec compartiments et tiroirs fermant à clé, en bois d'acajou orné d'une balustrade et autres ornements en or moulu et au-dessus de marbre blanc."

52. Ibid.: "Dans la salle à côté du salon d'hiver: sept tables de jeux dont une de brelan, deux de piques, deux de quadrille et une de tric-trac. "

53. Arch. dép. Aube, $144 \mathrm{~J} 40$ : l'inventaire révolutionnaire recense près de 3750 ouvrages. $22,6 \%$ des ouvrages relèvent de la littérature et $33,8 \%$ de l'histoire.

54. Le dessin appartient à une collection particulière. Il est reproduit dans l'article de Franck Gérard, "Le château de la Motte-Tilly et les travaux de 1780 ", op. cit., p. 11. 


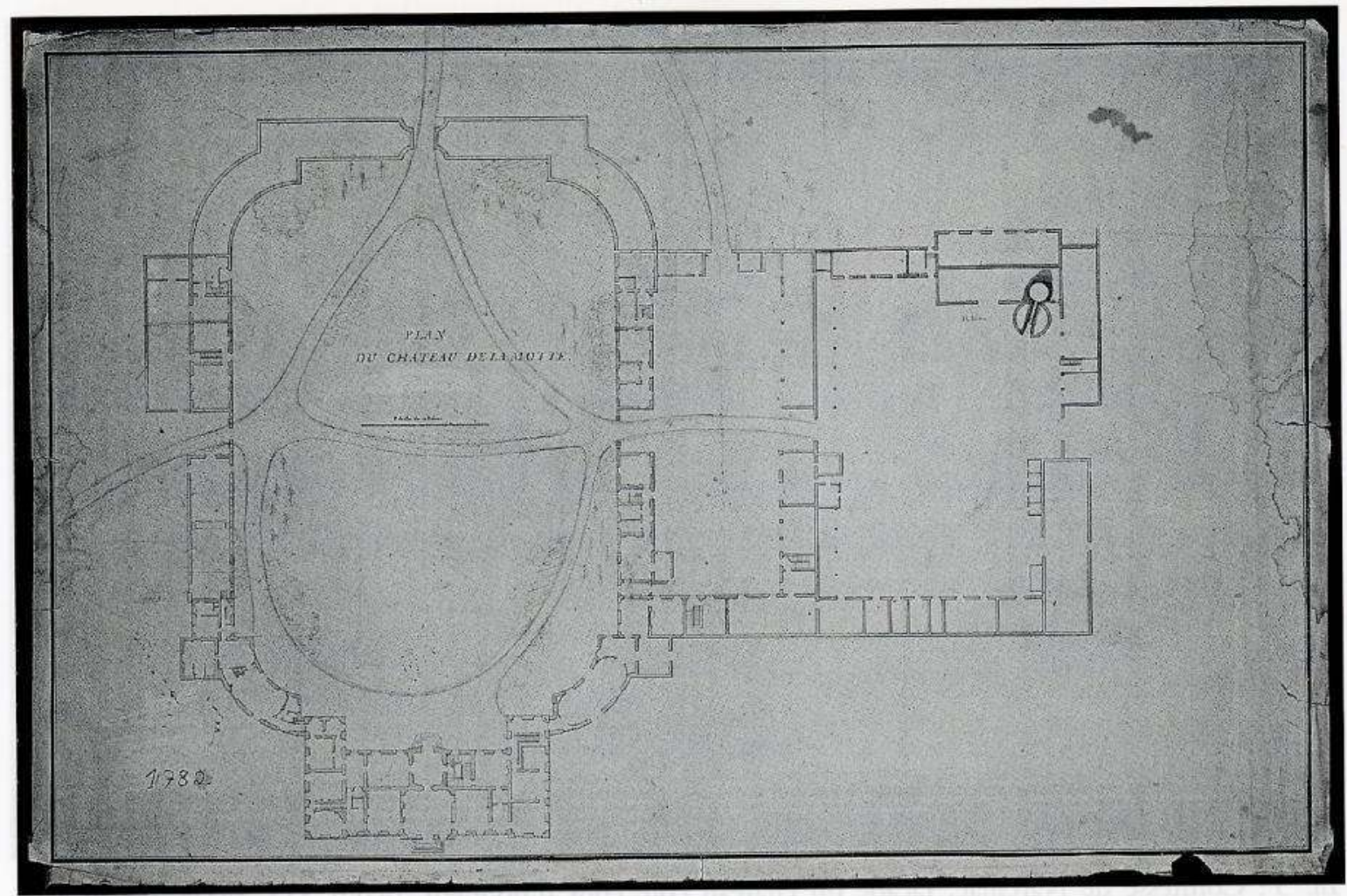

IIl. 4 : Anonyme "Plan du rez-de-chaussée du château et des dépendances de la Motte-Tilly ", 1782, dessin à la mine de plomb rehaussé à l'aquarelle, $150 \times 98 \mathrm{~cm}$. Arch. dép. de l'Aube, $144878 . \mathrm{Cl}$. de l'auteur.

rents documents iconographiques jusqu'à aujourd'hui montre que les façades n'ont pas subi de modification importante. Au début du XIXe siècle, la comtesse de Narcillac, résidente du château, réalise un dessin de la façade côté cour (ill. 5). Elle inscrit l'édifice dans un paysage bucolique. Ni les galeries de communication, ni les communs ne sont représentés; néanmoins la façade est très détaillée. Elle se compose d'un corps de bâtiment central avec un avant-corps qui forme un pavillon, prolongé par deux ailes en retour d'équerre. L'édifice se développe sur deux niveaux d'élévation et un niveau de comble. L'avant-corps central concentre les éléments d'ornements. L'architecte a joué avec la polychromie en usant de la pierre de taille pour la partie centrale et de la chaux pour le reste de la façade. Des portes-fenêtres sont percées et mises en valeur par un garde-corps au second niveau, au-dessus duquel prennent place les armoiries de la famille Terray, surmontées d'un arc segmentaire. La façade rend clairement compte de la distribution intérieure du château.

\section{La salle de spectacle}

La construction des salles de comédie privées s'étend depuis Paris aux châteaux de campagne dès le XVII ${ }^{e}$ siècle et perdure jusqu'au $\mathrm{XX}^{\mathrm{e}}$ siècle. Au XVIII ${ }^{e}$ siècle, le divertissement théâtral pouvait prendre place dans le salon, la galerie, l'orangerie ou encore dans les jardins ${ }^{55}$. Les théâtres permanents construits dans certains châ-

55. Marie-Emmanuelle Plagnol-Diéval, Les Théâtres de société : un autre théâtre ?, Paris, H. Champion, 2003, 324 p. 


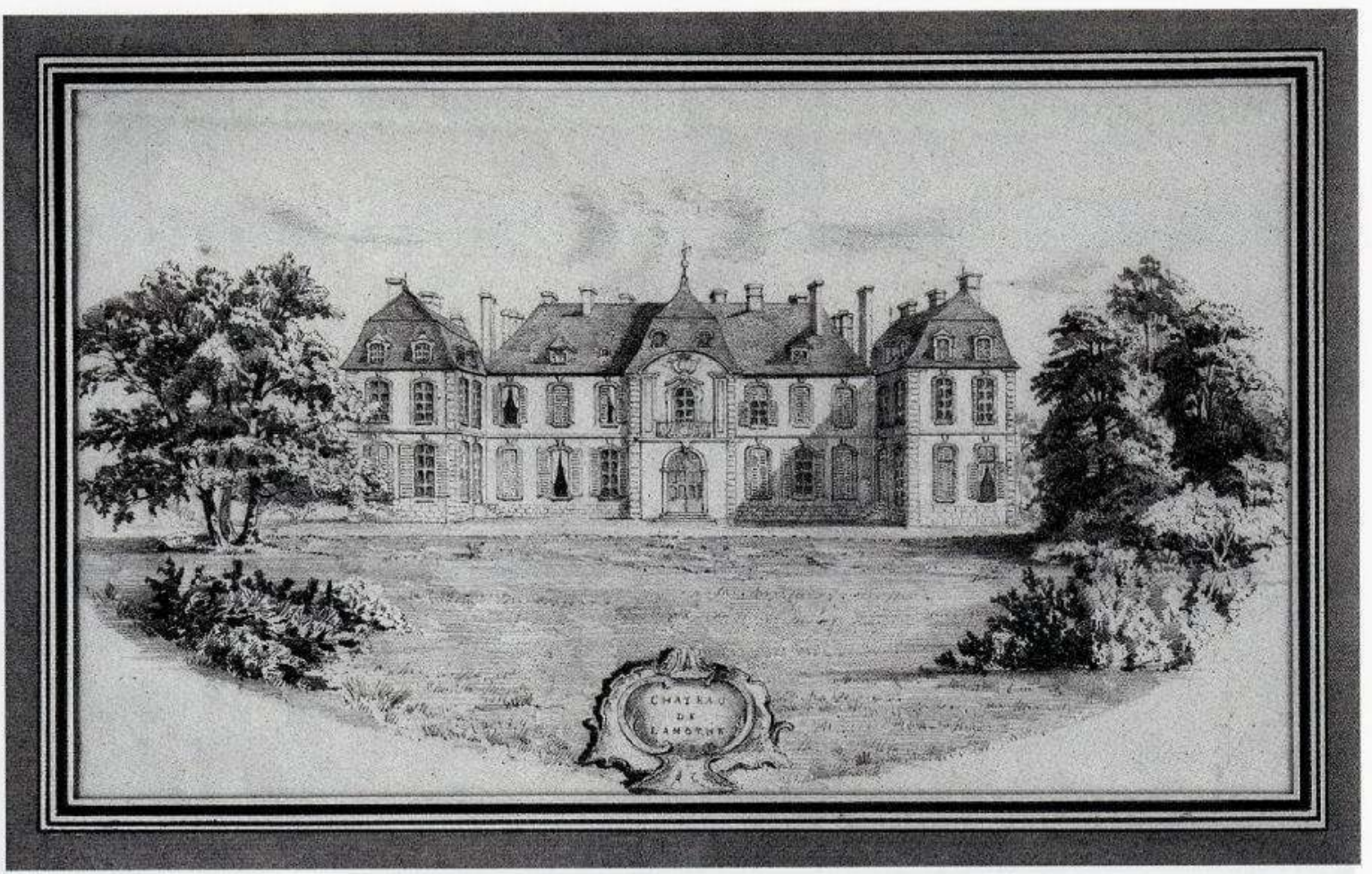

Ill. 5: Comtesse de Narcillac, façade du château côté cour d'honneur, début XIX ${ }^{e}$ siècle, dessin à la mine de plomb, collection du château de la Motte-Tilly. Cl. de l'auteur.

teaux pouvaient avoir d'autres fonctions, comme celles d'accueillir des mariages, des dîners ou des bals. Cet engouement se développe dans l'entourage d'une élite aisée et son importance est telle qu'il se développe au-delà de la sphère parisienne $^{56}$. La théâtromanie aurait également touché Joseph-Marie Terray et son frère Pierre Terray de Rosières. La salle de spectacle est édifiée dans les dépendances du château, côté est du bâtiment qui fait pendant à l'aile des communs, côté ouest (ill. 4). Pour y accéder, il fallait passer par une porte côté cour. Un seul plan existe, dont l'objet est l'épaisseur des murs de la pièce (ill. 6). Cette dernière s'organisait dans la longueur et la distribution se faisait en trois parties : le théâtre ou la scène, l'orchestre et le parterre. On peut noter la présence de deux escaliers de chaque côté de la scène qui permettaient de descendre sur le parterre; cela induit une scène en hauteur. La salle de spectacle n'est plus visible aujourd'hui. En effet, elle a été détruite par Claude Hippolyte Terray vers 1838 car elle demandait trop d'entretien. Elle n'avait sans doute plus le même intérêt qu'on lui portait au XVIII ${ }^{e}$ siècle. Néanmoins, les éléments connus nous permettent d'en avoir une idée. Deux plans datés de 1770 et de 1780 , croisés avec l'inventaire révolutionnaire, permettent de restituer la salle de spectacle. En 1794, Jacques Masson et Jean-Baptiste Anne Lorin, maçon et charpentier de Nogent-sur-Seine, visitent le

56. Antoine Lilti, "Public ou sociabilité ? Les théâtres de société au XVIII ${ }^{e}$ siècle ", Entre Renaissance et Lumières, Alain Viala, Christian Souhaud dir., Paris, Fayard, 2002, p. 281-300. 


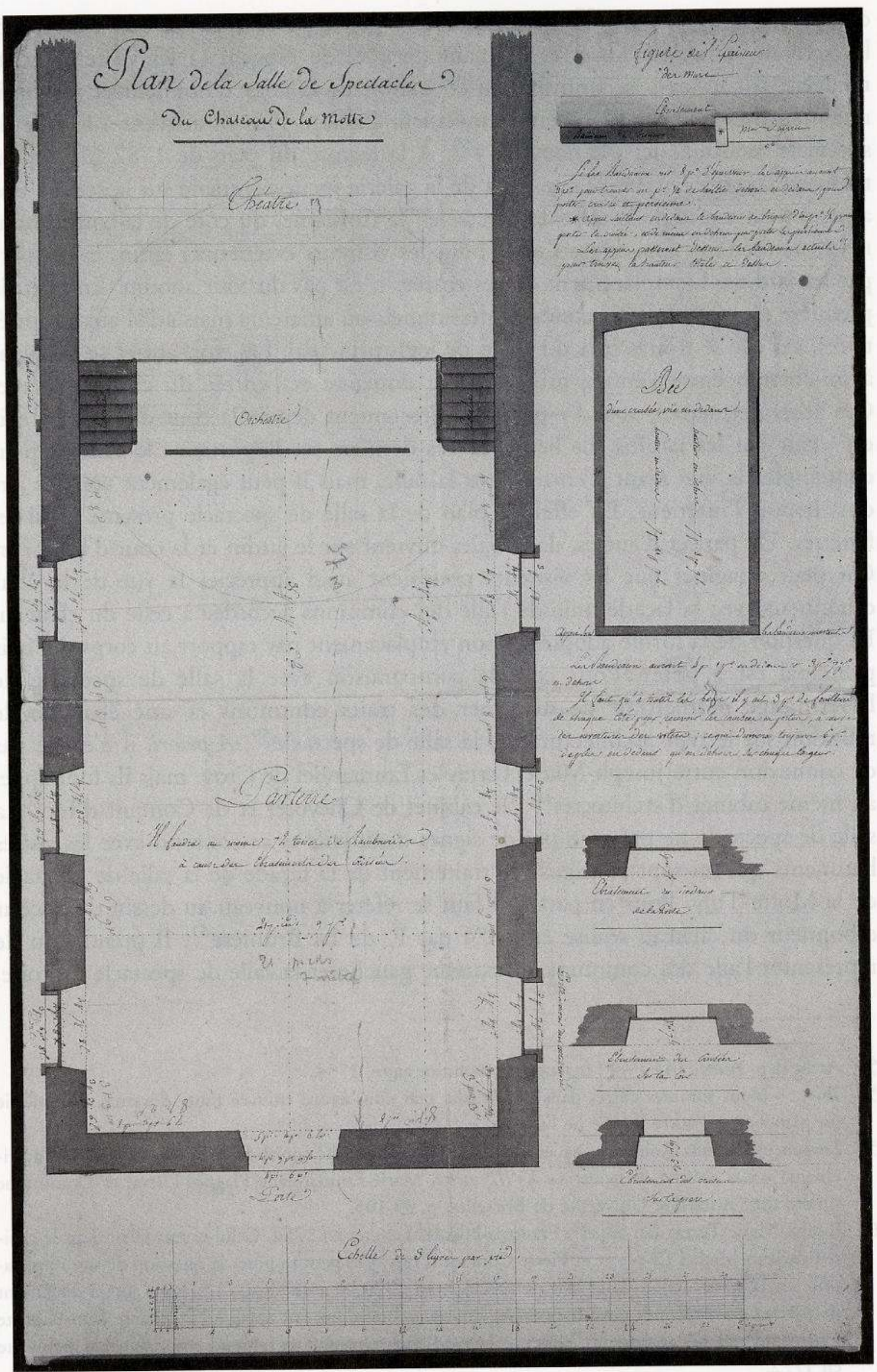

Ill. 6: Anonyme "Plan de la salle de spectacle du château de la Motte-Tilly ", [1770], mine de plomb et aquarelle, $75 \times 48 \mathrm{~cm}$. Arch. dép. de l'Aube, $144 \mathrm{~J} 877$. Cl. de l'auteur. 
château de la Motte-Tilly ${ }^{57}$. Ils commencent par le pavillon du garde "c'est-à-dire le pavillon contre les fossés et donnant du côté de Nogent " : les visiteurs commencent par les ailes se trouvant du côté droit de la cour d'honneur et ils continuent sur le logement d'un certain monsieur Baillon. Puis ils arrivent à la salle de spectacle, nommée ici "la comédie " 58 . A la lecture du plan de 1782 (ill. 4), on remarque quatre entrées : l'une, près de la galerie incurvée, mène sur la scène; une autre à l'extrémité de la salle de spectacle ; la troisième, du côté de la cour d'honneur, permettant sans doute l'accès pour les visiteurs extérieurs; enfin, la dernière par les jardins. L'emplacement de ces entrées n'est pas du tout anodin. En effet, la première est réservée aux acteurs professionnels ou amateurs mais aussi aux propriétaires, car elle se trouve près du corps de logis principal. Les trois autres se trouvent à mi-chemin entre l'entrée principale du domaine et l'entrée du château même. Ces accès permettent soit d'y pénétrer directement depuis la cour d'honneur, soit d'y venir par les jardins. Le lien avec ces derniers est important : le visiteur peut contempler la vue avant d'entrer dans la salle, mais il peut également voir les jardins depuis l'intérieur. En effet, le plan de la salle de spectacle présente aussi les fenêtres. De part et d'autres, deux baies ouvrent sur le jardin et la cour d'honneur. On peut imaginer que les visiteurs pouvaient aussi apprécier la vue de la cour d'honneur avec la façade unie de l'aile des communs accordée à celle du château. La question de la forme adoptée et son emplacement par rapport au corps de logis principal est à poser. Pour cela, la comparaison avec la salle de spectacle de l'Hermitage permettrait de discerner des traits communs et une ébauche de réflexion sur le type architectural de la salle de spectacle ${ }^{59}$. A priori, il n'existe pas de connexion entre Joseph-Marie Terray et Emmanuel de Croÿ, mais ils font appel au même cabinet d'architectes ${ }^{60}$ : le cabinet de Chevotet et de Contant d'Ivry. La salle de spectacle ne présente pas de signes distinctifs et se confond avec les autres bâtiments qui lui sont contigus : le traitement de la façade de la salle de spectacle de la Motte-Tilly. Pour en parler, il faut se référer à nouveau au dessin de la cour d'honneur du château réalisé en 1774 par F. de La Brunière ${ }^{61}$. Il prend soin de représenter l'aile des communs à l'extrême gauche et la salle de spectacle à droite,

57. Arch. dép. Aube, $144 \mathrm{~J} 40$ : inventaire révolutionnaire, 1794.

58. Ibid. : "Nous sommes entrés dans la comédie que nous avons trouvée toute dévastée, une moitié est planchée et l'autre moitié ne l'est pas."

59. Laurence Baudoux-Rousseau, "L'espace du théâtre de société (nord de la France et Pays-Bas autrichiens) ", Les théatres de société au XVIII siècle, Marie-Emmanuelle Plagnol-Diéval et Dominique Quéro dir., Bruxelles, Université de Bruxelles, p. 99-105.

60. Joseph-Marie Terray fait appel à François-Nicolas Lancret en 1754. Celui-ci travaillait dans le cabinet de Jean-Michel Chevotet et Pierre Contant d'Ivry. On peut se poser la question de son implication sur la construction de la salle de spectacle en 1770 bien que jusqu'à présent aucun document ne permet de confirmer cette hypothèse. Emmanuel de Croÿ fait appel à l'architecte Jean-Baptiste Chaussard qui fut également l'élève de Jean-Michel Chevotet et reprend son cabinet d'architecte en 1772 .

61. F. de La Brunière, [Cour d'honneur du château de la Motte-Tilly], dessin, dimensions inconnues, collection particulière. 
le château se trouvant au milieu de ces deux bâtiments et se distinguant par ses trois niveaux d'élévation et son toit brisé. Rien ne permet de distinguer le bâtiment des communs de la salle de spectacle. Le complexe architectural reporté par F. de La Brunière est uniforme. En comparant avec les plans de 1755 et de 1782, la surface réservée à la salle de spectacle respecte une symétrie rigoureuse et un rendu harmonieux de la cour d'honneur. Cet espace pouvait accueillir différents types d'événements en société : pièces de thêâtre, bals, festins... L'adaptation de cet espace s'explique par son emplacement en rez-de-chaussée permettant un accès direct depuis la cour d'honneur.

\section{Le premier état des jardins de 1755 à 1778}

Plusieurs éléments abordés par Jacques-François Blondel dans son traité sur les maisons de campagne ont été retenus dans la réalisation du château de la MotteTilly et de ses jardins. On note tout d'abord l'emplacement du corps de logis sur une éminence doublée d'une terrasse. Il s'agit de profiter de la vue mais également de l'air que l'on respire ${ }^{62}$. Ajouté à cela, cette disposition marque aussi l'expression du pouvoir du seigneur sur le territoire. Le théoricien ainsi que l'architecte souhaitent exprimer le plaisir de regarder le spectacle de la nature, mais aussi de marquer la présence du bâtiment dans le paysage : l'édifice doit être vu de loin par les voyageurs mais également par les habitants de la région ${ }^{63}$. Cela explique la volonté de mener à bien la destruction de l'ancien château qui se trouvait sur les bords de la Seine ${ }^{64}$. L'argument pour rattacher le château de la Motte-Tilly à la typologie de la maison de campagne réside dans le lien étroit établi entre le corps de logis et les jardins ${ }^{65}$. En effet, le rez-de-chaussée devient le "bel étage " qui permet un contact direct avec les jardins ${ }^{66}$. Ces derniers constituent un prolongement de la demeure et offrent, dans un cadre champêtre, les commodités et les agréments d'une vie sociale bien réglée. La jouissance de la nature se fait également par la promenade. Le jardin est donc ponctué de cabinets de verdure, de bosquets, de fabriques et d'autres agréments qui constituent autant de point de vue et de lieux de repos, voire d'endroits pour se protéger contre les éléments naturels. Le jardin élaboré du temps de l'abbé Terray n'existe plus aujourd'hui. De la même manière que les communs et la cour d'honneur, ils ont été régulièrement modifiés et le dernier état date des grands travaux du comte de Rohan-Chabot au début du

62. Jacques François Blondel, De la distribution des maisons de plaisance, op. cit., vol. 1, p. 8-9.

63. Katia Frey, La maison de campagne, programme idiomatique et universel, de Blondel à Ledoux (1737-1804), thèse de doctorat sous la direction de Daniel Rabreau, Université Paris I-Panthéon Sorbonne, 2002, p. 84.

64. Jean-Baptiste-Louis Cocquereau, Mémoires de l'abbé Terray, op. cit. : "Toutes lesdites terres et biens contigus l'un et l'autre ainsi qu'ils supervisent et comportent en l'état qu'ils sont actuellement, même les matériaux qui sont audit lieu de la Motte tel qu'il pourront se trouver provenant de la démolition du château. "

65. Jean-Baptiste-Louis Cocquereau, Mémoires de l'abbé Terray, op. cit., p. 88.

66. Ibid. 
$\mathrm{XX}^{\mathrm{e}}$ siècle ${ }^{67}$ réalisés à partir du plan de François-Nicolas Lancret de 1755. Ce plan ne montre pas l'intégralité du jardin. Pour cela, il faut regarder le plan du domaine réalisé avant les travaux réalisés par Antoine-Jean Terray, qui hérite des biens de son oncle en 1780. La présence du canal permettrait de dater ce plan aux alentours de 1770. Le plan massé des bâtiments en rose correspond au plan du toisé daté quant à lui de 1782. La ferme et les magasins sont représentés en bas à gauche du plan, qui feraient écho à la construction du canal puisque l'on constate la présence de quais et d'espace de stockage pour le blé. Le jardin s'apparente à une maison d'économie rustique décrite par Louis Ligier dans son CEconomie générale de la campagne, ou nouvelle maison rustique publiée en 1701 :

Il sera composé si l'on veut de parterre, embelli de toute sorte de fleurs, de belles allées de charmes, accompagné d'un grand potager, d'où l'on tirera une partie de la nourriture pour la maison. On n'oubliera pas au-dessous, d'y dresser un verger, afin de jouir du plaisir d'avoir beaucoup de fruits, qui est une douceur bien grande en quelque endroit qu'on puisse les rencontrer. De l'autre côté du verger se verra la pépinière et la bâtadière au-dessous [...]. Je présuppose que l'enceinte de toute cette maison sera de bonnes murailles, ou du moins de fortes haies vivres ${ }^{68}$.

La première terrasse est gravillonnée et peu profonde; elle est séparée de la seconde terrasse par un escalier de sept marches. Le jardin est séparé en son milieu par une longue allée, bordée de plates-bandes de gazon. Le dessin du parterre est composé de topiaires entourant un bassin. La pièce d'eau est quant à elle mise en évidence par des allées de tilleuls représentées dans le dessin au fusain de F. de La Brunière. Un aspect intéressant est le maintien de l'axe central qui se prolonge audelà de la Seine et des lieux-dits du Port-Saint-Nicolas et Villenauxe-la-Grande. Le plan présente quelques bosquets dont "le jardin des eaux " qui semble être fermé par une grille qui entoure la parcelle. À l'intérieur se trouve un plan d'eau circulaire. D'autres aménagements détaillés sont présents sur le plan. Certains sont marqués d'un numéro, induisant la présence d'une légende qui devait accompagner le plan, malheureusement perdue. L'espace se trouvant en bas à droite du plan semble être un cabinet de verdure dont une des entrées s'ouvre sur le canal. En effet, le bel étage qui ouvre sur le jardin met en avant la pièce d'eau et la Seine. Les principaux éléments d'ornementation sont les deux parterres se trouvant de part et d'autre de l'allée centrale. Les autres éléments de décoration et d'ornement se trouvent dans des espaces plus fermés et invite à la promenade et offre des accès dignes des honneurs du propriétaire. Le dessin de F. de La Brunière illustre également ce lien entre la nature et la sociabilité, en représentant des personnages au premier plan qui se promènent le long des allées de tilleuls et naviguent sur le

67. Arch. dép. Aube, 144 J 356 : "Les changements apportés par le comte de Rohan-Chabot au château de la Motte-Tilly en 1910 ", document imprimé et manuscrit, 1932.

68. Louis Ligier, Economie générale de la campagne, ou nouvelle maison rustique, Paris, Henri Desbordes, 1701, t. I, p. 13-14. 
canal. Les jardins sont légèrement en pente et le château se trouve au sommet de la composition. L'échelle des personnages représentés aux bords et sur le canal permet d'avoir une idée de l'étendue de ces jardins. Leurs activités illustrent la fonction du château : celle d'une maison de campagne. L'accession aux charges de contrôleur général des finances fut l'occasion de mettre en place des travaux d'agrandissement comme pour la salle de spectacle, mais également des travaux d'embellissement allant au-delà du domaine de la Motte-Tilly.

\section{L'illustration de l'ascension sociale de Joseph-Marie Terray?}

\section{Le canal Terray}

Le canal Terray avait un objectif très simple, celui de conduire les eaux de la Seine au château de la Motte-Tilly. En 1770, l'abbé tient l'autorisation du Conseil de faire creuser sur les pâtures de Nogent-sur-Seine un canal destiné à conduire l'eau sur le domaine ${ }^{69}$. Le seigneur avait également la charge de planter des arbres le long des berges de la tranchée, mais cela fut un échec puisque le chemin du canal n'était pas assez pentu. Les abords du canal Terray devinrent une promenade grâce aux plantations faites par Joseph-Marie Terray en $1771^{70}$. L'entreprise du contrôleur général des finances était sans doute de s'aligner avec les autres domaines à proximité, qui possédaient également un canal. Nous pouvons citer les canaux du château de Courtavant et de Ravois ${ }^{71}$. Ces canaux faisaient partie des axes fluviaux qui permettaient l'approvisionnement en foin et en avoine de la capitale ${ }^{72}$. Leur présence expliquerait peut-être la volonté de l'abbé d'en faire construire un pour le domaine de la Motte-Tilly. Il s'agit ęn effet d'un moyen de conduire l'eau dans les jardins, mais il est possible que cette entreprise visât une ambition plus grande. En effet sur le plan de 1780 et également sur un des plans du XIX ${ }^{e}$ siècle, des quais sont représentés ainsi que des magasins de stockage. L'ouvrage à charge de Jean-Baptiste-Louis Cocquereau, publié en 1777, tente de dénoncer les spéculations générales du contrôleur dans le commerce du grain :

69. Arch. dép. Aube, 144 J 990 : acte notarié passé à Nogent-sur-Seine entre Joseph-Marie Terray, Claude-Henri Laurenceau, conseiller du roi à l'élection de Nogent-sur-Seine, Louis Cransson et Étienne Lefebvre marchands et échevins à Nogent-sur-Seine. "Il sera loisible à mon dit seigneur abbé Terray de faire ouvrir un canal à l'embouchure de la rivière de la Seine au lieu-dit le Port aux charbons, de telle largeur et profondeur que l'on lui semblera et de faire passer à travers les pâtures de cette ville pour rejoindre les rivières de Roues-le-Roi, "

70. Ibid. : "À la charge de mon dit seigneur Terray de se faire construire un canal et de l'entretenir à ses frais [...] qu'il fera construire sur ledit canal au lieu-dit le Port aux charbons de largeur suffisante pour le charroi et le tirage des bateaux, de faire planter sur les deux bords et lancé dudit canal, les rangées d'arbres de chaque côté, convenable au terrain. "

71. Amédée Aufauvre, Histoire de Nogent-sur-Seine des temps anciens jusqu'à nos jours, Troyes, Bouquot, 1859 , p. 224

72. Ibid., p. 225. 
On a dit qu'en qualité de contrôleur général il avait touché plus de 450000 livres de pot de vin pour le bail des fermes renouvelé. Mais ce bail ne devant commencer qu'au 1 janvier 1775 [...]. En outre, sous prétexte des magasins du Roi, qu'il avait loués à sa terre de la Motte, aux compagnies chargées d'achat et d'emmagasinement des blés pour le compte de Sa Majesté, il avait fait paver une route magnifique depuis le grand chemin jusques chez lui, avec des ponts et quais, et cette dépense était évaluée de 4 à 500000 livres $^{73}$.

Dans ce texte, Jean-Baptiste-Louis Cocquereau rapporte des faits attribués au contrôleur général des finances dans la période précédant la guerre des farines du printemps 1775 . L'abbé aurait fait construire des routes, des canaux et des greniers à blés sur ses terres. Or des magasins sont bien décrits dans les inventaires révolutionnaires. Mais les archives relèvent plutôt chez l'abbé Terray un souci de s'occuper du domaine dans sa globalité et d'en augmenter la production ${ }^{74}$. Ainsi, les rapports annuels du domaine qui indiquent les dépenses et les recettes ne précisent pas la nature de ces dernières. Le régisseur du domaine, M. Bossuat, liste le montant et le nom de la personne qui effectue le paiement. Le dépouillement des documents relatifs à la gestion du domaine champenois a permis de comprendre la manière dont les terrains réservés à la culture des céréales et des fruits sont exploités. À cela s'ajoute la présence de grands magasins de blés dans les communs du château et de quatre moulins sur les domaines appartenant aux Terray.

L'hypothèse des magasins, décrits chez Jean-Baptiste-Louis Cocquereau comme ayant pour but de tirer profit et de spéculer sur le blé, ne tient pas. Les livres de comptes répertorient les entrées d'argent issues des banalités, des censives et des rentes que Joseph-Marie perçoit sur ses domaines. Ce canal devait avoir, au tout début de sa construction, une dimension économique, mais il fut réduit, par la suite, à l'alimentation en eau des jardins. Les documents conservés aux archives départementales de l'Aube contiennent également des plans d'arpentage, des actes notariés, de vente et d'échange de terres ainsi que des procès-verbaux entre Antoine-Jean Terray, neveu de l'abbé et propriétaire du domaine à partir de 1780 .

\section{Les chantiers de Joseph-Marie Terray}

Les chantiers initiés par Joseph-Marie Terray sont une manière d'ancrer la famille dans la région champenoise, mais aussi d'affirmer sa légitimité seigneuriale. Sa présence à la Motte-Tilly a été importante dès 1749 , mais elle s'amplifie à mesure que sa carrière prend de l'importance. Cela transparaît par les chantiers entrepris après sa nomination au poste de contrôleur général des finances. L'implication de l'abbé Terray dans la région se pose alors comme illustration de son importance dans l'administration de Louis XV ou bien de son attachement pour

73. Jean-Baptiste-Louis Cocquereau, Mémoires de l'abbé Terray, op. cit., p. 218.

74. Arch. dép. Aube, 144 J 767 : Livres de compte du domaine de la Motte-Tilly. Les revenus des domaines de Joseph-Marie Terray ont triplé sur quelques années. Parmi les domaines cités sont la Motte-Tilly, Courceroy, Gumery, Soligny, Avant, Rosières ainsi que le moulin de Chiénat. 
la région. En effet, dans son testament, l'abbé Terray exprime le souhait d'être inhumé à la Motte-Tilly ${ }^{75}$.

En poursuivant la lecture du testament de Joseph-Marie Terray, un legs attire l'attention : "Je donne et lègue à $\mathrm{M}$. Dumirat, mon neveu, ma terre de Béthon y compris les biens acquis de M. Dargentier et en outre la somme de 300000 livres pour l'aider à bâtir un château que j'ai souvent eu l'intention d'y faire construire ${ }^{76}$. " Ce domaine a été acquis par le contrôleur général en 1772 et se situe au nord de Nogent-sur-Seine entre Sézanne et Villenauxe-la-Grande. Or un projet de 1770, réalisé par Sieur Morla, géomètre des Ponts-et-chaussées ${ }^{77}$, a été mis au jour aux archives de la Marne, qui prévoyait une route pour relier Sézanne et Villenauxe. En regardant attentivement ce document, la route projetée passe par FontenayBéthon. Elle dessert également le château. Juste devant l'allée principale qui y conduit, une forme circulaire est réalisée, ce qui permettait de dégager cette allée et d'admirer l'édifice depuis la route. Cette piste n'a pas fait l'objet de recherches poussées, mais il faudrait poser la question de l'implication de Joseph-Marie Terray dans cette entreprise.

L'étude monographique du domaine de la Motte-Tilly offre l'occasion de prendre connaissance de ces archives récemment déposées aux archives départementales de l'Aube en 2005. L'étude apporte aussi de nouveaux éclairages sur l'abbé Joseph-Marie Terray, connu pour ses réformes sous le règne de Louis XV. En effet, le château de la Motte-Tilly fut édifié alors qu'il n'était encore que clerc au Parlement de Paris. Il s'agit ici de replacer l'édifice dans la carrière de l'abbé Terray. Le château se trouve être un élément clé dans l'ascension de son propriétaire, et constitue aussi une source de profits agricoles importants : on note la présence de terres réservées à la culture, des moulins, ainsi que des magasins destinés aux céréales. En outre, cette étude doit permettre d'apporter de nouvelles informations sur l'architecte qui a réalisé le plan du château, François-Nicolas Lancret. Sa participation à de nombreux projets dans la région de Champagne offre de nouvelles perspectives de recherches.

Rose-Marie CHAPALAIN doctorante à l'université de Paris-Ouest-La Défense

75. Arch. nat. M.C./XCIV/429 : dépôt du testament de M. l'abbé Terray, 22 février 1778 : "Je veux être enterré dans l'église de la Motte-Tilly, où je prie mon frère ou à défaut mon plus prochain héritier, de faire élever un mausolée dans la forme qu'il jugera convenable aux places que j'ai occupées. Il sera fondé un service annuel qui se célébrera dans cette église pour le repos de mon âme. "

76. Ibid.

77. Arch. dép. Marne, C 4031/27, $3^{\text {e }}$ feuillet: Carte pour servir au projet de la route de Sézanne à Nogent-sur-Seine, levée par le sieur Morla, 104,8 × 366,7 cm, 1770 . 